\title{
Convergence to a model in sparse-Lagrangian FDF simulations
}

\author{
A.Y. Klimenko and M.J. Cleary \\ The University of Queensland, \\ School of Mechanical and Mining Engineering, \\ Qld, 4072, Australia; \\ email: klimenko@mech.uq.edu.au \\ Published: Flow, Turbulence and Combustion, 85(3-4), pp. 567-591, 2010.
}

\begin{abstract}
This work investigates the problem of distinguishing modelling assumptions and numerical errors in sparseLagrangian FDF (Filtered Density Function) methods. A new interpretation of sparse modelling with Curl's mixing, which does not require an additional observation scale nor filtering, is given. The diffusion effects induced by mixing, which were previously interpreted as numerical errors, are now treated as modelling instruments. This ability of controlling numerical errors with the purpose of modelling physical quantities is one of the advantages of Lagrangian particle methods in turbulent reacting flows. The development of stochastic methods which use Lagrangian particles has been ongoing for many years, although the exact interpretation of the nature of such particles varies within the literature. Here we briefly discuss these interpretations and introduce the new term "Pope particles" - to unify terminology used for the particle simulations of turbulent reacting flows.
\end{abstract}

\section{Introduction}

The Filtered Density Function (FDF) methods [1-4] represent an adaptation of the Probability Density Function (PDF) approach [5] for LES (Large Eddy Simulations [6]) conditions. LES is based on the concept of resolving the largest scales in turbulent flows while the smallest (subfilter or subgrid) scales are modelled. In recent decades both LES and PDF methods have undergone impressive developments stimulated by an increase in computational 
power. In the framework of LES the distinction between numerical and modelling errors is not always clear [7]. It seems that the most commonly used form of LES, with a filtering operation indivisibly attached to numerical grid, does not represent a pure model nor a pure numerical method, but is a mixture of both. The FDF methods in general, and especially the sparse-Lagrangian FDF methods (discussed below), are linked to the LES methodology and hence in their applied form they too may represent mixture of a model and a numerical method. Finding an optimal interpretation for the modelling and numerical errors in sparse-Lagrangian simulations is the subject of the present work.

It is commonly argued that a proper scientific method should distinguish a model from its numerical implementation - this ensures repeatability and consistency of the simulations as well as adequate understanding of the model properties. A model that is inseparably linked to its numerics is likely to be sensitive to the particulars of a specific numerical implementation. Simulation results produced by these models can be difficult to reproduce consistently under changing computational conditions. Increased number of particles or grid points in simulations can be used differently in simulations. In the present work, we use the term "convergence to a model" to refer to the case when numerical errors become negligible, although, obviously, this type convergence does not eliminate modelling errors. Another type of convergence, brought about by increasing the number of grid points and/or Lagrangian particles in order to resolve all of the scales of turbulence is referred to as "convergence to DNS". In Ref.[8] these convergencies are studied as convergence with intensification and convergence with localisation. From an applied perspective, convergence to a model is computationally expensive and an engineer may be better off by reducing the overall error (i.e. modelling plus numerical errors) while keeping the computational cost relatively low. This approach, although reasonable and computationally efficient, results in a strong coupling between the model and the numerical scheme. The differences in simulation strategies noted above may be related to methodological differences between the disciplines of Science and Engineering [9]. Science is primarily concerned with a thorough understanding and consistent repeatability of the results while Engineering is more interested in their practical utility and affordability.

The FDF methods for simulating turbulent reacting flows reproduce the statistical distributions of the subfilter (or subgrid) quantities by stochastic modelling. The FDF methods (like the PDF methods from which they evolved) are formulated from a Lagrangian perspective and the use of stochastic particles brings significant advantages. The nature of these particles is discussed in detail in the next section. Typically, Lagrangian schemes permit a 
greater level of control over numerical diffusion than do Eulerian schemes. Perhaps the most important benefit of the Lagrangian FDF methods is that the chemical source terms, which are highly non-linear and sensitive to small variations in composition, are evaluated exactly. Despite these formidable advantages there is a large computational cost associated with FDF simulations. The emergence of sparse-Lagrangian FDF methods [10, 11], where the number of Lagrangian particles is much smaller than the number of Eulerian grid points, significantly reduces the computational cost associated with FDF methods; it even brings the cost below the cost of evaluating reactive scalars by Eulerian LES alone. The opposite case of having many particles per Eulerian cell is called intensive.

It is generally assumed that an insufficient number of particles or grid points will induce large numerical errors. As the number of particles is relatively small under sparse conditions, this inevitably brings questions about numerical accuracy and the separation of modelling and numerical errors. However, the Lagrangian FDF approach includes the option of utilising the stochastic fluctuations (which from a more conventional perspective would seen as numerical errors) as modelling tools. This conversion of a weakness into a strength is an inherent feature of the FDF methodology. Of course we do not rule out the possibility of interpreting the numerical errors in Eulerian LES in this FDF-like manner (i.e as fluctuating quantities modelling subgrid behaviour). In fact there are some reports that numerical errors may be used to partially offset modelling errors in conventional Eulerian LES $[12,13]$. If this is the case, the useful fraction of the errors have to be separated from the rest of the numerical method, then analysed and explicitly labelled as modelling quantities. Here this separation, analysis and labelling of is performed only for the sparse-Lagrangian FDF approach.

In the present work, we consider convergence to a model under conditions of a sparse distribution of particles. Our consideration is conceptual and performed for the case of constant density. The errors, which were previously seen as numerical errors, are now analysed and treated as modelling instruments. The issue of numerical convergence and accuracy in intensive FDF methods was previously analysed in Ref.[3, 4]. Convergence to DNS in sparse simulations was investigated in Ref.[8]. Our present interpretation of the sparse-Lagrangian methods differs from Ref.[14]: the present treatment is more direct, does not involve an additional observation scale and does not need explicit filtering of the simulated functions at that observation scale. 


\section{Pope particles}

Particles have traditionally played a prominent role in Fluid Mechanics. Fluid particles move with the local velocity of the fluid while Brownian particles not only move with the fluid velocity but are also engaged in a random walk, which continuously alters their positions and corresponds to the process of diffusion. The exact interpretation of the nature of these particles may vary. For example Brownian particles may be models for fluid molecules or small physical particles which are immersed in a flow, alternatively Brownian particles may be purely notional particles that are defined from a mathematical perspective. Fluid and Brownian particles do not possess scalar properties and can represent scalar concentrations only collectively by their number density.

The remarkable expansion of Lagrangian PDF methods during the last few decades is associated with another type of particles. The term "stochastic particle" was introduced in the review [5] which laid the foundations of the Lagrangian PDF methods. According to this review, the stochastic particles are interpreted as models for the motion of fluid particles and scalar values (i.e. the thermochemical composition of the fluid) evaluated at particle locations. The model is a Markov process which involves continuous movements in physical space and possibly discontinuous mixing between particles. The particle distributions are determined in a numerically efficient way by solving stochastic differential equations. The governing PDF equation for stochastic particles involves both Fokker-Planck and Poisson-type terms [5]. The parameters of the stochastic process are selected to consistently match the PDFs of the particles with the PDFs of the physical scalars and velocities in the turbulent flow.

The subsequent development of Lagrangian methods has resulted in the evolution of that original stochastic particle concept. Application to boundary layer conditions required the introduction of particles whose position in physical space is subject to Brownian fluctuations [15]. Stochastic particles became a part of hybrid methods that combine Lagrangian stochastic simulations of the PDF of reacting scalars with Eulerian solutions of the Navier-Stokes equation [16]. The hybrid FDF approach [3,4] adapts the concept of this hybrid PDF models for LES conditions. The particles, which are used in the stochastic formulation of the FDF concept [3], follow the LES resolved large-scale motions in physical space and model the subgrid fluctuations. The emergence of sparse-Lagrangian FDF methods $[10,11]$ resulted in consideration of sparse particle systems that are moved on the background of Eulerian velocity fields that are much better (or fully) resolved. Under these conditions, stochastic particles follow the trajectories of Brownian or fluid particles and may serve not only as models but also as tools

of direct numerical simulation (DNS) of scalar transport [8]. The development of hybrid methods has resulted 
not only in the convergence of PDF, RANS and LES/DNS but also in the synergy of PDF methods and models based on the use of the mixture fraction — namely the Flamelet [17, 18] and CMC [19] methods. Particles used in the MMC approach [20], which effectively unifies PDF and CMC methodologies, are traced in an extension of the physical space by MMC reference variables [10]. We refer to this combined physical/reference space as the extended physical space.

The previous paragraphs illustrate the great breadth of use of stochastic particles since their conceptualisation. It should be noted that the term "stochastic particles" was not universally accepted beyond the area of Fluid Mechanics despite the cross-disciplinary use of this term, to refer to particles of any kind that exhibit some sort of stochastic behaviour. Even in the literature dedicated to stochastic modelling of reacting flows, the use of the term "stochastic particles" is often mixed up with other similar terms. The subsequent major reviews and books on modelling of reacting flows [17, 21-26] use various terms (Lagrangian particles, notional particles, stochastic trajectories, etc) to denote essentially the same thing — the stochastic particles introduced by Pope [5]. In publications on atmospheric dispersion, the term "marked particles" is reserved for notional particles without properties or mixing while the stochastic particles with properties and mixing are used without any specific term being assigned to them [27]. The existing terminological uncertainty indicates the need for a more general name for particles used in stochastic simulations of reacting and non-reacting flows that can encompass and unify the different terms. Therefore, we suggest the term "Pope particles", which is accurately defined below, and believe that this definition directly addresses the need. It is also intended that the term "Pope particles" will serve as an acknowledgment of the outstanding contribution of S.B. Pope to Science and Engineering and especially to his crucial role in introducing the methodology and establishing the principal framework for stochastic Lagrangian modelling of reacting flows. It is expected that the term "Pope particles" will receive cross-disciplinary recognition and that interpretations of Pope particles will continue to evolve in line with future developments in the area.

Pope particles are used to simulate a variety of properties in turbulent reacting flows. Unlike marked particles which are fully characterised by their locations, Pope particles have a set of properties and values of these properties are assigned to, or modelled by, each of the particles. These properties include scalars and, possibly, velocities and other flow parameters needed in simulations. Since fluid velocity and scalars are continuous fields when considered from the Eulerian perspective, the assigned properties are understood as the values of these fields evaluated at the location of the particles. 
We now define Pope particles as notional particles with assigned properties that

1. model or follow the trajectories of fluid particles, Brownian particles or molecules in physical or extended physical space;

2. model the assigned properties with the assistance of a mixing model;

3. in reacting flows evolve due to chemical reactions.

The essential and distinctive features of Pope particles are that they have a set of assigned property values and a mixing operation is performed on them which affects the values of those properties. Mixing may involve various forms of direct exchanges between particles or interactions with mean values. The mixing models that are commonly used in Lagrangian simulations (Curl's and modified Curl's models, IEM, EMST, MMC and others) are repeatedly reviewed in publications $[5,17,21-26]$.

In the present work we use Pope particles in the context of hybrid sparse-Lagrangian FDF/LES methods. The particles have a certain PDF which, with the appropriate selection of parameters, is a model for the FDF. Each particle is representative of the PDF, but due to the low number of particles used in sparse simulations we may not be able to evaluate that PDF locally and instantaneously. Irrespective of our ability to evaluate it the particle PDF exists whether we know its distribution or not. Statistical quantities of interest (i.e. moments of the PDF) can be accumulated locally over a long period of time or instantaneously over a larger volume containing a greater number of particles. Alternatively, many particles can be used under conditions of convergence to a model to populate the simulations and visualize the PDF both locally and instantaneously. Obviously, this latter option imposes a significant computational cost.

The random walk of particles $p=1, \ldots, n$ with scalar properties and mixing is represented by the following stochastic Ito equations

$$
\begin{gathered}
d x_{p}^{i}=A^{i}\left(\mathbf{x}_{p}, t\right) d t+b^{i j}\left(\mathbf{x}_{p}, t\right) d \omega_{p}^{j} \\
\frac{d Z_{p}^{(\alpha)}}{d t}=W_{p}^{(\alpha)}+\left[\frac{d Z_{p}^{(\alpha)}}{d t}\right]_{\text {mix }}
\end{gathered}
$$

Here $x_{p}^{i}$ is $i^{\text {th }}$ physical coordinate of particle $p$ and $Z_{p}^{(\alpha)}$ represents the scalar properties of this particle. The summation convention over repeated indices applies here and further in the paper. The particle PDF is given by $P(\mathbf{Z}, \mathbf{x} ; t)=P_{Z}(\mathbf{Z} \mid \mathbf{x} ; t) P_{x}(\mathbf{x} ; t)$ where $P_{x}$ is linked to particle number density $\rho_{n}$ and the overall number of 
particles $n$ by $P_{x}=\rho_{n} / n$. Both $P_{x}$ and $\rho_{n}$ are constant in a constant density flow. The PDF $P_{Z}$ is evaluated for a given realisation of the velocity field and governed by a modified Fokker-Planck equation

$$
\frac{\partial P_{Z}}{\partial t}+\frac{\partial A^{i} P_{Z}}{\partial x^{i}}-\frac{\partial^{2} B^{i j} P_{Z}}{\partial x^{i} \partial x^{j}}+\frac{\partial W^{(\alpha)} P_{Z}}{\partial Z^{(\alpha)}}=\left[\frac{d P_{Z}}{d t}\right]_{\text {mix }}
$$

Here, $\omega_{p}^{j}$ represent Wiener processes, $\mathbf{Z}=\left\{Z^{(1)}, \ldots Z^{\left(n_{Z}\right)}\right\}$ are simulated scalar values assigned to the particles, $W=W(\mathbf{Z})$ is the chemical source term, $A^{i}$ is the drift coefficient linked to fluid velocity, $B^{i j}=b^{i l} b^{j l} / 2$ is the diffusion coefficient, and $[\cdot]_{\text {mix }}$ represents the mixing operation. Note that notation $\left[d Z^{(\alpha)} / d t\right]_{\text {mix }}$ is symbolic and does not necessarily imply that conventional derivatives $d Z^{(\alpha)} / d t$ exist. The specific forms of the model parameters are discussed further in the remaining sections.

\section{Convergence to a model in sparse-Lagrangian simulations}

The number of Pope particles under sparse conditions is much smaller than the number of Eulerian grid points. Hence, the velocity field evaluated on the Eulerian gird is much better resolved than the reacting scalars evaluated on the particles. In our present consideration, we assume that a fully resolved velocity field is available in the simulations; that is the Pope particles are transported within a DNS-simulated velocity field. That assumption, rather than the more practical case of a filtered velocity field by LES, is a conceptual simplification that avoids the added confusion of there being several (Lagrangian and Eulerian) filter scales. We consider the case of constant density, which is another simplifying assumption. In practical applications, sparse-Lagrangian simulations are coupled to an LES-simulated velocity field and the molecular diffusion coefficient in the FDF transport equation is replaced by a turbulent diffusion coefficient associated with the Eulerian LES filter scale.

Convergence to a model implies increasing the number of Pope particles to a very large value in a way that reduces numerical error but does not significantly alter the substance of the model. As discussed above, this type of convergence is not particularly useful from a practical perspective - many particles produce modelling effects which are similar to those for relatively few particles and furthermore convergence to a model would be difficult to achieve due to practical limitations on computing resources. A larger number of particles can be more efficiently used to progressively resolve the finer turbulent scales and effectively approach the DNS limit. This convergence of Lagrangian simulations to DNS was analysed in Ref.[8] and is not considered further in the present work. From a theoretical perspective, however, convergence of a numerical scheme to a model permits a thorough 
characterisation of that model. For sparse simulations an analysis of this type of convergence removes the main conceptual difficulty — although properties of the flow exist locally and instantaneously those properties are known accurately only at a fairly sparse set of particles and evaluation of all local and instantaneous properties is possible only if the observation scales are increased or more particles are added.

The sparse particle distribution effectively applies a filtering operation on the field as explained below. The fluctuations at the sub-Lagrangian-grid (or Lagrangian filter) scale are modelled by the fluctuations of the particle values $z=Z-\langle Z\rangle$. In this section, we consider a selected scalar and the index " $\alpha$ " is omitted for clarity. In the stochastic differential equations (1) and (2) governing the Lagrangian FDF the mixing operator is the only term which involves an interaction between particles (either directly or via a mean) and the remaining spatial transport and chemical reaction terms are applied to individual particles in isolation. Therefore it is only the mixing operation which is affected by the spacing between particles and hence affected the number of particles used in the simulations. The practices employed in sparse simulations [11], when very few particles are present, is to select particles for mixing which are closest to each other in the extended physical space. This space is defined as the product of physical space and MMC reference space. We denote the characteristic mixing distance in physical space as $r_{m}$. For sparse simulation conditions $r_{m}$ coincides with the characteristic distance between the mixing particles. The characteristic time of the mixing operation should be selected in compliance with the relationships of the inertial interval

$$
\tau_{\text {mix }} \equiv \frac{\left\langle z^{2}\right\rangle}{\bar{N}} \sim \tau_{m} \equiv\left(\frac{r_{m}^{2}}{\bar{\varepsilon}}\right)^{1 / 3}
$$

where $\bar{N}$ and $\bar{\varepsilon}$ are the filtered averages of scalar dissipation and dissipation of kinetic energy, respectively. Here, the angular brackets denote averages over the ensemble of realisations of the stochastic processes $\omega_{p}^{j}$ while the filtering operation is introduced in the following section. The sparse model does not resolve scalar fields at distances $r \leq r_{m}$.

Mixing between particles separated by a finite distance generates a substantial diffusion which was called "numerical diffusion" in previous publications $[8,28]$. In the present work, this diffusion will be interpreted differently and is referred to as mixing-induced diffusion. As the number of Pope particles increases, we have two major options: I) to reduce the mixing distance; or II) to keep the mixing distance approximately the same. In case I we keep $\tau_{\text {mix }}$ unchanged from its value for sparse conditions; indeed, if we reduce $\tau_{\text {mix }}$, this would enforce a new model, similar to the original sparse model but with a higher resolution. Progressive refining of the resolution 
corresponds to convergence to DNS and is not of interest here. Reducing the mixing distance while keeping $\tau_{\text {mix }}$ the same will result in inconsistent modelling unless the model is modified in some other way — mixing induced diffusion is negligible under conditions of complete localization and $\langle Z\rangle$ represents a fully resolved scalar field but since $\tau_{\text {mix }}$ is not reduced an excessive variance $\left\langle(z)^{2}\right\rangle=\bar{N} \tau_{\text {mix }}$ will be present in the simulations. Thus consistent modeling for case I requires the introduction of a modified turbulent diffusion associated with the scale $r_{m}$ and defined by

$$
D_{t} \sim\left(\bar{\varepsilon} r_{m}^{4}\right)^{1 / 3}=\frac{r_{m}^{2}}{\tau_{m}}
$$

The turbulent diffusion removes the smallest fluctuations from $\langle Z\rangle$ and these are emulated instead by the fluctuations $z$. As shown in the next section the modification required for case $\mathrm{I}$ is achieved by selecting $\mathbf{A}=\overline{\mathbf{U}}$ and using $B=D+D_{t}$. The small-scale fluctuations induced by $\mathbf{u}=\mathbf{U}-\overline{\mathbf{U}}$ are now modelled by the turbulent diffusion $D_{t}$ and setting $A=\mathbf{U}$ instead would result in an excessive level of small-scale random particle motions. (As previously noted, we assume that fully resolved velocity fields are available in the simulations.)

The other approach, case II, preserves the original mixing length given by $r_{m}$ although this length is not linked any more to the distance between the particles. This means that within a distance of $r_{m}$ of a given particle there are many particles of which any one can be selected randomly for mixing. In this case, mixing-induced diffusion with coefficient $D_{m}$ plays the role of turbulent diffusion. This convergence case is the closest of the two cases to the conditions observed under the (original) sparse simulation conditions. The differences to the original conditions are related to variations of distances between the particles. Indeed, under the original sparse conditions, mixing couples are formed by the closest particles. The PDF of a distance between closest particles can be easily determined as follows. Since positions of randomly walking particles are statistically independent from each other, the probability of having no other particles in a volume $V_{r}$ around a given particle is given by

$$
\mathbb{P}\left(n_{V_{r}}=0\right)=\left(1-\frac{V_{r}}{V_{n}}\right)^{n}=\left(1-\rho_{n} \frac{V_{r}}{n}\right)^{n} \underset{n \gg 1}{\longrightarrow} \exp \left(-\rho_{n} V_{r}\right)
$$

Here, $\rho_{n}$ is the number density of particles, $V_{n}=n / \rho_{n}$ is the effective volume of the domain containing $n$ particles and we assume that the number of particles $n$ is large so that $n-1 \approx n$. The volume, $V_{r}$, depends on the parameter $r$ which can be selected as the physical distance between particles or in any other suitable way. The distribution of distances between closest particles, which is linked to the probability of having no other particles within volume 
$V_{r}$ is given by

$$
P_{r}=\left|\frac{\partial \mathbb{P}\left(n_{V_{r}}=0\right)}{\partial r}\right|=\frac{\partial V_{r}}{\partial r} \rho_{n} \exp \left(-\rho_{n} V_{r}\right)
$$

If we increase the number of particles and specify mixing vicinity by condition $r \leq r_{m}$, the distribution of distances between particles is subject to changes. Since the model for case II is close to the original sparse model the $\rightarrow$ differences between two such simulations are interpreted as numerical errors according to our present considerations. As noted previously, the numerical errors do not always represent a negative feature of the simulations. In principle, particle mixing pairs in a case II simulation can be selected in accordance with the PDF $P_{r}$ given by (7). However, as the magnitude of the characteristic distance between mixing particles has a far more significant impact on simulations than does the PDF of this distance, such a selection algorithm seems unnecessary.

To summarise, we consider two limiting approaches for convergence to a model

Case I) subfilter turbulent diffusion is modelled by particle random walk and

Case II) subfilter turbulent diffusion is modelled by mixing-induced diffusion (previously called "numerical diffusion")

Case II is closes to the conditions in the original sparse simulations while the modelling strategy of case I is closer to conventional (intensive) simulations.

\section{Modelling the FDF equation}

For the reactive scalars $Y^{(\alpha)}$ which are governed by the equation

$$
\frac{\partial Y^{(\alpha)}}{\partial t}+\frac{\partial Y^{(\alpha)} U^{i}}{\partial x^{i}}-D_{0} \frac{\partial^{2} Y^{(\alpha)}}{\partial x^{i} \partial x^{i}}=W^{(\alpha)}
$$

the FDF transport equation is given by $[3,4]$

$$
\frac{\partial \bar{F}}{\partial t}+\nabla \cdot\left(\overline{\mathbf{U}}_{Y} \bar{F}\right)+\frac{\partial\left(W^{(\alpha)}+D_{0}{\overline{\left(\nabla Y^{(\alpha)}\right.}}_{Y}\right) \bar{F}}{\partial Y^{(\alpha)}}=0
$$

where

$$
F \equiv \delta\left(\mathbf{Y}-\mathbf{Y}^{\circ}\right)
$$

is the fine grained distribution, whose filtered average $\bar{F}$ represents an FDF. In the above a sum is evaluated over repeated indicies. The variable $\mathbf{Y}^{\circ}$ is the sample space variable for $\mathbf{Y}$, although we omit superscript "o" when the 
use of sample space variables is obvious from the context. The subscript " $Y$ " is used to denote filtering conditional on $Y$

$$
\bar{\varphi}_{Y}=\frac{\overline{\varphi F}}{\bar{F}}
$$

for any $\varphi$. The filtered quantities are introduced by applying the following filtering operation

$$
\bar{\varphi}(\mathbf{x})=\int_{\infty} \varphi\left(\mathbf{x}^{\circ}\right) \Psi_{r}\left(\mathbf{x}-\mathbf{x}^{\circ}\right) d x^{\circ}
$$

to any fully resolved field $\varphi(\mathbf{x})$ where the filter kernel $\Psi_{r}$ is characterized by the filtering scale $r$. As differential diffusion effects are neglected here, the FDF governing equation can be rewritten in the form

$$
\frac{\partial \bar{F}}{\partial t}+\nabla \cdot\left(\overline{\mathbf{U}}_{Y} \bar{F}\right)+\frac{\partial W^{(\alpha)} \bar{F}}{\partial Y^{(\alpha)}}=D_{0} \nabla^{2} \bar{F}-\frac{\partial^{2} \bar{N}_{Y}^{(\alpha \beta)} \bar{F}}{\partial Y^{(\alpha)} \partial Y^{(\beta)}}
$$

where $N^{(\alpha \beta)}=D_{0} \nabla Y^{(\alpha)} \cdot \nabla Y^{(\beta)}$ is the dissipation tensor and $D_{0}$ is assumed to be constant.

\subsection{Model for Case I dominated by the random walk}

The random walk model (case I) simulates subfilter turbulent diffusion by the random walk of Pope particles and presumes the following approximations

$$
A^{i}=\bar{U}^{i}(\mathbf{x}, t)+\frac{\partial B}{\partial x^{i}}, \quad B^{i j}=\delta^{i j} B, \quad B=D_{t}+D_{0}
$$

where $D_{t}$ is the effective turbulent diffusion coefficient for subfilter fluctuations. The PDF equation (3) takes the form

$$
\frac{\partial P_{Z}}{\partial t}+\nabla \cdot\left(\overline{\mathbf{U}} P_{Z}\right)+\frac{\partial W^{(\alpha)} P_{Z}}{\partial Z^{(\alpha)}}=\nabla \cdot\left(B \nabla\left(P_{Z}\right)\right)+\left[\frac{d P_{Z}}{d t}\right]_{\text {mix } 0}
$$

Here, we use the index "mix0" to stress that mixing is fully localized (i.e. performed between particles located at distances $r \rightarrow 0$ ). The specific form of mixing in this equation is not important for matching the FDF equation as long as mixing is compliant with the well-known mixing constraints $[3,4]$. Here, we stress two of these constraints

$$
\int\left[\frac{d P_{Z}}{d t}\right]_{\operatorname{mix} 0} d \mathbf{Z}=0
$$

and

$$
\int Z^{(\alpha)}\left[\frac{d P_{Z}}{d t}\right]_{\operatorname{mix} 0} d \mathbf{Z}=\left[\frac{d\left\langle Z^{(\alpha)}\right\rangle}{d t}\right]_{\operatorname{mix} 0}=0 .
$$

One can see that equation (15) is a consistent model for equation (13) with the following modelling approximations

$$
-\overline{\mathbf{u}}_{Y} \bar{F} \simeq D_{t} \nabla P_{Z}
$$


and

$$
-\frac{\partial^{2} \bar{N}_{Y}^{(\alpha \beta)} \bar{F}}{\partial Y^{(\alpha)} \partial Y^{(\beta)}} \simeq\left[\frac{d P_{Z}}{d t}\right]_{\operatorname{mix} 0}
$$

where $\overline{\mathbf{u}}_{Y} \equiv \overline{\mathbf{U}}_{Y}-\overline{\mathbf{U}}$ and the symbol " $\simeq$ " means "is modeled by". These approximations are conventional in FDF modelling [3, 4, 25, 29].

\subsection{Model for Case II with diffusion generated by mixing}

In this case the subfilter turbulent diffusion is simulated using diffusion induced by mixing, which is not fully localized. Unlike in Ref.[14], we do not apply any explicit filtering operation to $P_{Z}$ while implicit filtering is effectively performed by non-local mixing effects. The scales smaller than the mixing distance, which is assumed to belong to the inertial interval of turbulence, can be called "submixing" scales in accordance with conventional LES terminology. Surrounding each particle is a mixing volume or mixing vicinity from which mixing partners for that particle are selected. The size of mixing volume is determined by mixing distance. The modelling approximations depend significantly on the type of mixing used in the simulations. In this work, we consider only Curl's mixing [30] (or modified Curl's mixing [31]) which can be specified by the following equations for mixing of any two particles denoted here as particles " 1 " and "2" [8]

$$
\begin{gathered}
\mathbf{Z}_{1}=\mathbf{Z}_{1}^{\circ}+\tilde{\eta}\left(\mathbf{Z}_{2}^{\circ}-\mathbf{Z}_{1}^{\circ}\right) \\
{\left[\frac{d P_{Z}\left(Z \mid \mathbf{x}_{1}\right)}{d t}\right]_{\text {mix }}=(n-1) P_{x} \int \theta_{12}\left[P_{Z}\left(Z \mid \mathbf{x}_{1}\right) * P_{Z}\left(Z \mid \mathbf{x}_{2}\right)\right]_{\text {mix }} d \mathbf{x}_{2}} \\
{\left[P_{1}\left(Z_{1} \mid \mathbf{x}_{1}\right) * P_{2}\left(Z_{1} \mid \mathbf{x}_{2}\right)\right]_{\text {mix }} \equiv \int P_{t}\left(Z_{1} \mid Z_{1}^{\circ}, Z_{2}^{\circ}\right) P_{1}\left(Z_{1}^{\circ} \mid \mathbf{x}_{1}\right) P_{2}\left(Z_{2}^{\circ} \mid \mathbf{x}_{2}\right) d Z_{1}^{\circ} d Z_{2}^{\circ}}
\end{gathered}
$$

where the superscript "o" is used to denote the values before mixing, parameter $\theta$ determines intensity of mixing

as discussed in Ref. [8], $P_{t}$ is transitional probability, and a new operator $\left[P_{1} * P_{2}\right]_{\text {mix }}$ has been introduced for convenience of notation. We presume that the extent of mixing $\eta$ is randomly selected as suggested in modified Curl's model [31]. As previously noted, the particle values are treated as being statistically independent; that is $P\left(Z_{1}, \mathbf{x}_{1}, Z_{2}, \mathbf{x}_{2}\right)=P_{Z}\left(Z_{1}, \mathbf{x}_{1}\right) P_{Z}\left(Z_{2}, \mathbf{x}_{2}\right)$. We also use Bayes theorem $P\left(Z_{1}^{\circ}, \mathbf{x}_{1}\right)=P_{Z}\left(Z_{1}^{\circ} \mid \mathbf{x}_{1}\right) P_{x}$ and note that

$$
P_{x}\left[\frac{d P_{Z}\left(Z \mid \mathbf{x}_{1}\right)}{d t}\right]_{\text {mix }}=\left[\frac{d P\left(Z, \mathbf{x}_{1}\right)}{d t}\right]_{\text {mix }}=(n-1) \int \theta_{12}\left[P\left(Z, \mathbf{x}_{1}\right) * P\left(Z, \mathbf{x}_{2}\right)\right]_{\text {mix }} d \mathbf{x}_{2} .
$$

As shown in the Appendix, the mixing operator can be represented by the following expansion

$$
\left[\frac{d P_{Z}}{d t}\right]_{\text {mix }}=\left[\frac{d P_{Z}}{d t}\right]_{\text {mix } 0}+\left[\frac{d P_{Z}}{d t}\right]_{\text {mix } 2 \mathrm{D}}+\left[\frac{d P_{Z}}{d t}\right]_{\operatorname{mix} 2 \mathrm{~N}}+\ldots
$$


where "mix0" denotes a fully localized mixing compliant with the constraint (17), "mix2D" denotes the diffusive effect of mixing and "mix $2 \mathrm{~N}$ " denotes a diffusion-related generation or dissipation term

$$
\begin{gathered}
{\left[\frac{d P_{Z}}{d t}\right]_{\text {mix } 0}=\hat{\theta}\left[P_{Z} * P_{Z}\right]_{\text {mix }}} \\
{\left[\frac{d P_{Z}}{d t}\right]_{\text {mix } 2 \mathrm{D}}=\nabla \cdot\left(\frac{\hat{\theta} I}{2}\left[P_{Z} * \nabla P_{Z}\right]_{\text {mix }}\right)} \\
{\left[\frac{d P_{Z}}{d t}\right]_{\text {mix } 2 \mathrm{~N}}=-\frac{\hat{\theta} I}{2}\left[\nabla P_{Z} * \nabla P_{Z}\right]_{\text {mix }}}
\end{gathered}
$$

where $I=\hat{r}_{1}^{2}$ is dependent on the radius of the mixing volume which is assumed to be spherical with $\hat{r}_{1} \sim r_{m}$. A more complete set of equations that presented above can be found in the Appendix and also Ref. [8]). All of these terms are compliant with constraint (16). The term "mix2D" is diffusive and changes the mean values, $\left\langle Z^{(\alpha)}\right\rangle$, while the term "mix2N" satisfies (17) and represents a generation of variance due to non-localness of mixing. Existence of this term can be illustrated by applying a Curl's mixing operation to a field with $z=0$ and a constant gradient $\nabla Z=$ const. Mixing that is not fully localized results in appearance of some variance $z^{2}>0$ due to exchanges between particles with different values of $Z$.

With the following selection of the parameters

$$
\mathbf{A}=\overline{\mathbf{U}}(\mathbf{x}, t), B=D_{0}
$$

where formula for $A^{i}$ does not involve gradients of density $\rho$ and molecular diffusivity $D_{0}$ since these values are assumed constant. The PDF equation now takes the form

$$
\begin{aligned}
& \frac{\partial P_{Z}}{\partial t}+\nabla \cdot(\left(\mathbf{A} P_{Z}\right)+\frac{\partial W^{(\alpha)} P_{Z}}{\partial Z^{(\alpha)}}=\left[\frac{d P_{Z}}{d t}\right]_{\operatorname{mix} 0}+\left[\frac{d P_{Z}}{d t}\right]_{\text {mix } 2 \mathrm{~N}}+ \\
&+\nabla \cdot\left(B \nabla P_{Z}+\frac{\hat{\theta} I}{2}\left[P_{Z} * \nabla P_{Z}\right]_{\text {mix }}\right)
\end{aligned}
$$

This equation is consistent with the exact FDF equation (i.e. $P_{Z} \simeq \bar{F}$ ) by making the following modelling approximations:

$$
\begin{aligned}
-\overline{\mathbf{u}}_{Y} \bar{F} & \simeq \frac{\hat{\theta} I}{2}\left[P_{Z} * \nabla P_{Z}\right]_{\mathrm{mix}} \\
-\frac{\partial^{2} \bar{N}_{Y}^{(\alpha \beta)} \bar{F}}{\partial Y^{(\alpha)} \partial Y^{(\beta)}} & \simeq\left[\frac{d P_{Z}}{d t}\right]_{\operatorname{mix} 0}+\left[\frac{d P_{Z}}{d t}\right]_{\operatorname{mix} 2 \mathrm{~N}}
\end{aligned}
$$

We note that the modelled turbulent transport terms of (25) appear in the FDF/PDF equations in a consistent divergent form (see discussion in the appendix) and that the modelled terms of (26) preserve mean values ( $\bar{Y}$ and $\langle Z\rangle)$. 
Another issue relevant to convergence to a model that needs to be mentioned here is generation of conditional fluctuations by the mixing operators - the performance of Curl's mixing in this respect is dependent on the details of the mixing scheme. As the number of Pope particles increases, generation of conditional fluctuations by Curl's mixing needs to be controlled. Generation of conditional fluctuations was specifically analysed in Ref.[32] and is not further considered here.

\section{Equations for the scalar mean and variance}

In this section we investigate how the two models (cases I and II) perform in simulating transport of means and variances. For the sake of simplicity, the index " $\alpha$ " is again omitted although equations are valid for any of the scalars $\alpha=1, \ldots, n_{Z}$ The equations for the first and second moments can be derived from the FDF equation as

$$
\frac{\partial \bar{Y}}{\partial t}+\nabla \cdot(\overline{\mathbf{U Y}})-D_{0} \nabla^{2} \bar{Y}=\bar{W}
$$

and

$$
\frac{\partial \overline{Y^{2}}}{\partial t}+\nabla \cdot\left(\overline{\mathbf{U} Y^{2}}\right)-D_{0} \nabla^{2} \overline{Y^{2}}=2 \overline{W Y}-2 D_{0} \overline{(\nabla Y)^{2}}
$$

The equation for the variance can be obtained from the moment equations as

$$
\frac{\partial\left\langle y^{2}\right\rangle_{\bar{F}}}{\partial t}+\nabla \cdot\left(\left\langle\mathbf{U} y^{2}\right\rangle_{\bar{F}}\right)-D_{0} \nabla^{2}\left\langle y^{2}\right\rangle_{\bar{F}}=2\langle w y\rangle_{\bar{F}}-2 \nabla \bar{Y} \cdot\langle\mathbf{u} y\rangle_{\bar{F}}-2 D_{0}\left(\overline{(\nabla Y)^{2}}-(\nabla \bar{Y})^{2}\right)
$$

were $y=Y-\bar{Y}$ and the angular brackets indicate averaging by integration with the FDF [33, 34], for example

$$
\left\langle\mathbf{U} y^{2}\right\rangle_{\bar{F}}=\int(Y-\bar{Y})^{2} \overline{\mathbf{U}}_{Y} \bar{F}(Y) d Y
$$

Although $\langle Y\rangle=\bar{Y}$, this is not necessarily valid for other quantities. For example, $\langle y\rangle=0$ but $\bar{y} \neq 0$. Note that some of the quantities (e.g. $\overline{(\nabla Y)^{2}}$ ) may be not defined in terms of $\bar{F}(Y)$.

The corresponding moment and variance equations for the models are given by

$$
\begin{gathered}
\frac{\partial\langle Z\rangle}{\partial t}+\nabla \cdot(\tilde{\mathbf{A}}\langle Z\rangle)-\nabla(B \nabla\langle Z\rangle)=\langle W\rangle+\left[\frac{d\langle Z\rangle}{d t}\right]_{\text {mix }} \\
\frac{\partial\left\langle Z^{2}\right\rangle}{\partial t}+\nabla \cdot\left(\tilde{\mathbf{A}}\left\langle Z^{2}\right\rangle\right)-\nabla\left(B \nabla\left\langle Z^{2}\right\rangle\right)=2\langle W Z\rangle+\left[\frac{d\left\langle Z^{2}\right\rangle}{d t}\right]_{\text {mix }} \\
\frac{\partial\left\langle z^{2}\right\rangle}{\partial t}+\nabla \cdot\left(\tilde{\mathbf{A}}\left\langle z^{2}\right\rangle\right)-\nabla\left(B \nabla\left\langle z^{2}\right\rangle\right)=2 B(\nabla\langle Z\rangle)^{2}+2\langle w z\rangle+\left[\frac{d\left\langle z^{2}\right\rangle}{d t}\right]_{\text {mix }}
\end{gathered}
$$

where we introduce $\tilde{\mathbf{A}}=\mathbf{A}-\nabla B$. 


\subsection{Mean and variance for model case I}

In the model case I dominated by the random walk we obtain

$$
\begin{gathered}
\frac{\partial\langle Z\rangle}{\partial t}+\nabla \cdot(\overline{\mathbf{U}}\langle Z\rangle)-\nabla(B \nabla\langle Z\rangle)=\langle W\rangle \\
\frac{\partial\left\langle z^{2}\right\rangle}{\partial t}+\nabla \cdot\left(\overline{\mathbf{U}}\left\langle z^{2}\right\rangle\right)-\nabla\left(B \nabla\left\langle z^{2}\right\rangle\right)=2 B(\nabla\langle Z\rangle)^{2}+2\langle w z\rangle-2 \frac{\left\langle z^{2}\right\rangle}{\tau_{\text {mix }}}
\end{gathered}
$$

where

$$
\overline{\mathbf{U}}=\mathbf{A}-\nabla B=\tilde{\mathbf{A}}, \quad B=D_{t}+D_{0}, \quad \tau_{\text {mix }} \equiv \frac{1}{\langle\tilde{\gamma}\rangle \hat{\theta}}, \quad \tilde{\gamma} \equiv \tilde{\eta}-\tilde{\eta}^{2}
$$

Where $\hat{\theta}$ is overall intensity of mixing as specified in the Appendix and Ref. [8]. It is easy to see that consistency of the model and the transport equations is based on the following modelling approximations

$$
\begin{gathered}
\langle\mathbf{u} y\rangle \simeq-D_{t} \nabla\langle Z\rangle \\
\left\langle\mathbf{u} y^{2}\right\rangle \simeq-D_{t} \nabla\left\langle z^{2}\right\rangle \\
D_{0} \overline{(\nabla Y)^{2}} \simeq \frac{\left\langle z^{2}\right\rangle}{\tau_{\text {mix }}}
\end{gathered}
$$

These approximations are conventional in LES and FDF modelling.

\subsection{Mean and variance for model case II}

Equations for the moments of Curl's mixing operator accounting for non-local mixing effects are derived in the Appendix. With the assumption that mixing volume is spherical such that $I^{i j}=\delta^{i j} I$ then the equations for the mean and variance take the form

$$
\begin{gathered}
\frac{\partial\langle Z\rangle}{\partial t}+\nabla \cdot(\mathbf{A}\langle Z\rangle)-\nabla\left(\left(B+D_{m}^{(1)}\right) \nabla\langle Z\rangle\right)=\langle W\rangle \\
\frac{\partial\left\langle z^{2}\right\rangle}{\partial t}+\nabla \cdot\left(\mathbf{A}\left\langle z^{2}\right\rangle\right)-\nabla\left(\left(B+D_{m}^{(2)}\right) \nabla\left\langle z^{2}\right\rangle\right)=2 B(\nabla\langle Z\rangle)^{2}+2\langle w z\rangle-2 \frac{\left\langle z^{2}\right\rangle+G}{\tau_{\text {mix }}}+2 D_{m}^{(1)}(\nabla\langle Z\rangle)^{2}
\end{gathered}
$$

where for consistency with the moments of the FDF transport equation the model parameters are given by

$$
\begin{gathered}
\mathbf{A}=\overline{\mathbf{U}}, \quad B=D_{0}, \quad \tau_{\text {mix }}=\frac{1}{\langle\tilde{\gamma}\rangle \hat{\theta}}, \quad \tilde{\gamma}=\tilde{\eta}-\tilde{\eta}^{2} \\
D_{m}^{(1)}=\frac{\hat{\theta} I\langle\tilde{\eta}\rangle}{2}, \quad D_{m}^{(2)}=\frac{\hat{\theta} I\left\langle\tilde{\eta}^{2}\right\rangle}{2}, I=\hat{r}_{1}^{2}, \quad G=\frac{I}{2}(\nabla\langle Z\rangle)^{2}
\end{gathered}
$$


Note that $D_{0}$ is assumed constant. The derived equations are consistent with scalar transport equations with the following modelling approximations

$$
\begin{gathered}
\langle\mathbf{u} y\rangle_{\bar{F}} \simeq-D_{m}^{(1)} \nabla\langle Z\rangle \\
\left\langle\mathbf{u} y^{2}\right\rangle_{\bar{F}} \simeq-D_{m}^{(2)} \nabla\left\langle z^{2}\right\rangle \\
D_{0} \overline{(\nabla Y)^{2}} \simeq \frac{\left\langle z^{2}\right\rangle+G}{\tau_{\text {mix }}}
\end{gathered}
$$

It can be seen that, unlike in case I, the transport coefficients for the two moments can be different, although both coefficients $D_{m}^{(1)}$ and $D_{m}^{(2)}$ have the correct magnitude of $\sim \hat{r}_{1}^{2} / \tau_{\text {mix }}$ provided that $\tau_{\text {mix }}$ is selected according to (4). The additional dissipation term $G / \tau_{\text {mix }}$ in the above is related to the fact that variance within the mixing volume is greater than $\left\langle z^{2}\right\rangle$ due to changes in the scalar mean $\langle Z\rangle$ within this volume. Indeed, the dissipation rate is determined by the difference $Z_{2}-Z_{1}$ averaged over the mixing volume

$$
G_{2} \equiv\left\langle\left(Z_{2}-Z_{1}\right)^{2}\right\rangle_{V}=\left\langle z_{1}^{2}\right\rangle+\left\langle z_{2}^{2}\right\rangle_{V}+\left\langle\left(\left\langle Z_{2}\right\rangle-\left\langle Z_{1}\right\rangle\right)^{2}\right\rangle_{V} \approx 2\left(\left\langle z^{2}\right\rangle+G\right)
$$

The variance $G_{2}$ exceeds $2\left\langle z^{2}\right\rangle$ by $2 G$ inducing additional dissipation within the mixing volume.

The coefficient $\mathbf{A}$ is selected in (41) as the filtered velocity $\overline{\mathbf{U}}$. However velocity filtering is not recommended for highly sparse conditions. Indeed, putting $\mathbf{A}=\overline{\mathbf{U}}$ would restrict the random stirring of particles by subfilter turbulent fluctuations to the level corresponding to molecular diffusion and the particles would be forced to repeatedly form the same mixing couples. This would increase stochastic dependencies between particle values. This is not a problem when many particles are present in the mixing volume and couples are stirred up by random selection of mixing partners. The particles can, of course, be stirred up by increasing $B$ and intensifying the random walk. This, however, introduces additional diffusion and corresponds to an effective combination of model cases I and II. Velocity filtering may also represent a problem for MMC mixing as considered in the next section.

Replacing $\mathbf{A}=\overline{\mathbf{U}}$ by $\mathbf{A}=\mathbf{U}$ in equations (24), (39) and (40) results in the appearance of subfilter frequencies induced by $\mathbf{u}=\mathbf{U}-\overline{\mathbf{U}}$. The influence of these frequencies on the scalar field is small due to the dominating influence of mixing-induced diffusion over modelled scalar fields at these scales. In the diffusion-dominated inertial region the spectrum of scalar fluctuations decays rapidly $\left(\sim k^{-17 / 3}\right.$ according to Batchelor et. al.[35], where $k$ is the wave vector), although not exponentially. These fluctuations, although small and not detectable under truly sparse conditions (highly sparse simulations reproduce equivalent statistics but not small-scale structures of scalar fields), affect the value of $\langle Z\rangle$ but can not be interpreted as resolved components. The interpretation of these vari- 
ations depends on the definition of the converged model: they represent numerical errors if $\mathbf{A}=\overline{\mathbf{U}}$ at the limit of $n \rightarrow \infty$. These errors (interpreted as numerical) can be eliminated by progressive filtering of the velocity field as the number of Pope particles $n$ increases and the constraint related to independence of particles vanishes. Independence of particles can be practically ensured by A) having a sufficient random component in particle motions or by B) having a sufficiently large number of particles in the mixing vicinity[8]. Complying with condition B makes enforcing condition A unnecessary: under these conditions filtering out high-frequency components of velocity does not affect independence of particles.

The present analysis of the sparse model results in conclusions that may seem paradoxical: mixing-induced diffusion previously considered as a numerical error is treated as a modelling quantity while fully resolved highfrequency oscillations of velocity are used for numerical purposes (of stirring particles without generating additional diffusion) and induce numerical errors. It is possible, of course, to filter equation (24) and assume that $\bar{F} \simeq \bar{P}[14]$ but this is not the interpretation considered in the present work.

\section{Remarks on MMC in sparse-Lagrangian simulations}

In general, sparse-Lagrangian simulations may work with different mixing models but so far only MMC-Curl has been demonstrated to work under sparse conditions[10, 11]. Multiple Mapping Conditioning or MMC [20] is one of the tools that can ensure a high quality of simulations while using only relatively few Pope particles. Although MMC may involve many reference (i.e. conditioning) variables, here we consider only a single MMC reference variable - the DNS-evaluated (Eulerian) mixture fraction $f \equiv Y_{f}$. Note that the simulations involve another mixture fraction $Z_{f}-$ a Lagrangian quantity assigned to Pope particles.

In the original MMC [20], the reference variables were represented by continuous Markov processes, while in generalised MMC [32], only some of the reference variables represented by a Markov family are used for localisation (conditioning) of the mixing operation. Once we accept generalised MMC, we can use any stochastic process to simulate reference variables as any (generally non-Markovian) process can be approximated well by a Markov family of a much larger dimension. For example, it is arguable point that Lagrangian particle trajectories in a turbulent flow are not Markovian, while tracing these particles with the use of LES or DNS is a Markov family of a very large dimension, which is determined by the total number of parameters at every grid point and at every 
particle location. Thus, it is legitimate to consider evaluation of the mixture fraction and velocities by LES or DNS supplemented by a particle tracing algorithm as means of creating reference stochastic process for proper localisation of mixing. The goal of these simulations is to obtain a reference mixture fraction which is as close to real mixture fraction as possible. This interpretation corresponds to interpreting generalised MMC as a PDF method with LES/DNS used simply for producing good reference variables, which are used to improve simulation of mixing. In this case, the values carried by Pope particles are expected to match the joint PDFs of reacting scalars but are not required to reproduce filtered fields. The present work gives an alternative interpretation for MMCLES/DNS as an FDF method with a Lagrangian filtering scale determined under sparse conditions by distance between particles, presuming that this filter may be quite coarse compared to the Eulerian LES filter but still has a reasonable resolution (i.e. the Lagrangian filtering scale belongs to the inertial interval of turbulence).

Application of MMC principles becomes more transparent under conditions of convergence to the model. Since the mixing volume $V_{p}$ of a particle $p$ contains many particles under these conditions, a mixing partner for particle $p$ can be selected among these particles indiscriminately or, if MMC ideas are employed, with preference given to particles that have the same (or close) value of $f$ as the particle $p$. The scale of the mixing volume $r_{m}$ determines the filtering scale but, in accordance with MMC principles[11, 20,36], the mixing time $\tau_{\text {mix }}$ is now selected as

$$
\tau_{\text {mix }}=c \tau_{m}
$$

and with $c<1$ is smaller than that specified by equation (4). Indeed, in MMC we distinguish minor and major variations of $Z_{f}$ in the mixing volume $G_{V}=G_{\mathrm{mj}}+G_{\mathrm{mn}}[20,36]$ where $G_{V}=\left\langle\left(Z_{f}-\left\langle Z_{f}\right\rangle_{V}\right)^{2}\right\rangle_{V}$ is the overall variation of $Z_{f}$ within the mixing volume, and $G_{\mathrm{mj}}=\left\langle\left(\bar{Z}_{f}-\left\langle Z_{f}\right\rangle_{V}\right)^{2}\right\rangle_{V}, \bar{Z}_{f}=\left\langle Z_{f} \mid f\right\rangle_{V}$ and $G_{\mathrm{mn}}=$ $\left\langle\left(Z_{f}-\bar{Z}_{f}\right)^{2}\right\rangle_{V}$ are the major and minor variations, respectively. In major variations, $Z_{f}$ fluctuates jointly with $f$ while minor variations of $Z_{f}$ are independent of $f$. The MMC mixing dissipates directly only minor variations and $\bar{N}_{f}=G_{\mathrm{mn}} / \tau_{\text {mix }}$ while, if MMC is not used, the dissipation is determined by overall variations $\bar{N}_{f}=G_{V} / \tau_{\text {mix }}$. Since $G_{\mathrm{mn}}<G_{V}$ and the dissipation rate is a macro-parameter and should be model-invariant, the value of $\tau_{\text {mix }}$ must be smaller under MMC conditions and $c<1$. The condition of having minor dissipation time $\tau_{\text {mix }}$ being essentially smaller than the overall characteristic dissipation time $\tau_{m}$ is common for MMC models [36].

The MMC constraint on mixing can be expressed by defining the effective distance $d_{p q}$ between particles $p$ 
and $q$

$$
d_{p q}^{2}=\sum_{i=1,2,3} \frac{\left(x_{i}^{(q)}-x_{i}^{(p)}\right)^{2}}{r_{i}^{2}}+\frac{\left(f^{(q)}-f^{(p)}\right)^{2}}{f_{m}^{2}}
$$

that depends on the selection of characteristic scales $r_{1}, r_{2}, r_{3}$ and $f_{m}$. This means that instead of selecting particles with a given value of $f$ in a spherical mixing volume, one can define a stretched mixing volume such that all particles within this volume have close values of $f$. Mixing between particles $p$ and $q$ is allowed (i.e. $\theta_{p q}>0$ ) if $d_{p q}^{2} \leq d_{0}^{2}$ where $d_{0} \sim 1$. Although selecting different physical scales in different directions can be beneficial for MMC mixing, we select here $r_{1}=r_{2}=r_{3}=r_{m}$. The MMC mixing has another scale $r_{f}=\left(\bar{\varepsilon} / \bar{N}_{f}^{3}\right)^{1 / 2} f_{m}^{3}$ associated with the process and the parameters $r_{m}$ and $f_{m}$ are selected so that $r_{f}$ is noticeably smaller than $r_{m}$ enforcing close proximity of mixing couples in the mixture fraction space. The volume of mixing vicinity can be estimated by $V_{m}=r_{m}^{2}\left(r_{m} / r_{f}\right)^{2 / 3} r_{f} \sim r_{m}^{8 / 3} r_{f}^{1 / 3} \sim\left(\bar{\varepsilon} / \bar{N}_{f}^{3}\right)^{1 / 6} r_{m}^{8 / 3} f_{m}$ assuming that the surfaces of $f=$ const are fractals having the dimension of $8 / 3[17,37]$.

The MMC mixing volume has two characteristic scales $r_{m}$ and $r_{f}$ and, consequently, two characteristic coefficients for mixing-induced diffusion $D_{m} \sim r_{m}^{2} / \tau_{\text {mix }}$ and $D_{f} \sim r_{f}^{2} / \tau_{\text {mix }}$ are associated with these scales. Here, we denote $D_{m}$ as an estimation of $D_{m}^{(1)}, D_{m}^{(2)}$ and other mixing-related diffusion coefficients. We note that if $\tau_{\text {mix }}<\tau_{m}$ then $r_{f}^{2}$ can not be larger than $r_{f}^{2} \sim \bar{\varepsilon} \tau_{\text {mix }}^{3}$ (that is $f_{m}^{2}$ can not be larger than $f_{m}^{2} \sim \bar{N}_{f} \tau_{\text {mix }}$ ), otherwise the diffusion $D_{f}$ would be disproportionally large and generate excessive diffusion in $f$-space (according to CMC [19], the correct value of the diffusion coefficient in $f$-space is $\bar{N}_{f}$ ). At the same time, selecting $f_{m}^{2}$ much smaller than $\bar{N}_{f} \tau_{\text {mix }}$ does not improve localisation in the composition space due to presence of minor fluctuations $G_{\mathrm{mn}} \approx \bar{N}_{f} \tau_{\text {mix }}$ but, as for any other grid refinement, it does requires additional computational resources. Hence, the optimal choice of $f_{m}$ should be in line with the following estimation $f_{m}^{2} \sim \bar{N}_{f} \tau_{\text {mix }}$. There is, however, a side-effect of reducing $\tau_{\text {mix }}$ from $\tau_{m}$ to $c \tau_{m}$ and that is the corresponding increase in the diffusion coefficient $D_{m}$ from $\sim r_{m}^{2} / \tau_{m}$ to $\sim c^{-1} r_{m}^{2} / \tau_{m}$. The main spatial effect of the mixing operation on the model is determined by the mixing-induced diffusion coefficients and we may wish to keep these coefficients the same as $\tau_{\text {mix }}$ decreases. This requires using a new scale $r_{m}^{\prime}=c^{1 / 2} r_{m}$ as the scale defining the mixing vicinity; the filtering scale though remains $r_{m}$. Note that this reduction is not possible under the original sparse conditions when the mixing volume on average contains only a single particle. Improvement in the resolution in $f$-space results in an increase in $r_{m}$ and a decreased resolution in other (less important) directions.

Under truly sparse conditions, there is only one partner particle within the mixing volume surrounding a 
given particle; $V_{m} \sim 1 / n$. We also note the mixing volume estimate $V_{m} \sim r_{m}^{8 / 3} f_{m}$, which was derived previously in this section. If the number of particles changes from $n_{1}$ to $n_{2}$, we may wish, or if $n_{2}<n_{1}$ be forced, to keep simulations sparse but preserve the original simulating conditions as much as it is possible. Since these conditions are highly dependent on the treatment of the mixture fraction, we assume $f_{m 2}=f_{m 1}$. Hence $\left(r_{m 2} / r_{m 1}\right)^{8 / 3}=V_{m 2} / V_{m 1}=n_{1} / n_{2}$ or for the parameter $r_{m} / f_{m}$, which determines the strength of mixturefraction conditioning in MMC simulations, we obtain $r_{m} / f_{m} \sim n^{-3 / 8}$. This equation represents an estimate for the expected optimal dependence of the MMC conditioning parameter on the number of particles used in sparse simulations. This estimate is obtained under specific assumptions given above and needs further investigation but the trend of increasing $r_{m} / f_{m}$ with decrease in $n$ should be noted.

In many practical cases the relatively higher value of diffusion along the surfaces of $f=$ const would not have a significant impact on the quality of the simulations as long as the simulations are well-resolved in $f$-space. In principle, there could be cases when diffusion $D_{m}$ is excessive and its effect is detrimental: for example high levels of extinction may need a stricter control over diffusional exchanges between burning and extinct areas (although we have not observed these difficulties in simulations of the flames $\mathrm{D}$ and $\mathrm{E}$ that we performed so far $[10,11,41])$. Possible solutions may involve an increase in the number of Pope particles and/or the introduction of doubleconditioning[38], which requires a second reference variable in order to minimise minor fluctuations. This second reference variable must be indicative of the locations of extinction zones and their transport by turbulence, while also possessing a significant degree of independence from particle scalar values.

It is noted that using filtered velocity may present a problem for MMC-LES simulations. Indeed, the scales $\sim r_{f}$ of velocity components across iso-scalar surfaces need to be resolved in order to accurately represent the process of diffusion in $f$-space while a rougher resolution of $\sim r_{m}$ can be sufficient for other directions. In principle, it is possible to tackle this problem by introducing highly non-isotropic, flow-dependent filtering; $\Psi_{r}(\mathbf{x})$ in (12) could be stretched along the surfaces of $f=$ const. But filtering velocity field in this way is complicated. It seems that leaving velocity without Lagrangian-scale filtering is a more practical solution, which is used in actual sparse MMC-LES simulations [11] where the Eulerian filter scale is much smaller than the Lagrangian filter scale. As previously noted, our analysis assumes that DNS-generated velocity fields are available to avoid the confusion of having two filtering scales (Lagrangian and Eulerian). Our analysis remains similar for LES but velocity $\mathbf{U}$ must replaced by $\overline{\mathbf{U}}_{E}$ and molecular diffusivity $D_{0}$ must be replaced by turbulent diffusivity $D_{0}+D_{E t}$ where the 
subscript " $E$ " is used to emphasise that this filtering and this turbulent diffusivity are associated with the Eulerian filtering scale. The scalar transport equation (8) is now replaced by

$$
\frac{\partial \bar{Y}_{E}^{(\alpha)}}{\partial t}+\frac{\partial \bar{Y}_{E}^{(\alpha)} \bar{U}^{i}}{\partial x^{i}}-\frac{\partial}{\partial x^{i}}\left(\left(D_{0}+D_{E t}\right) \frac{\partial \bar{Y}_{E}^{(\alpha)}}{\partial x^{i}}\right)=\bar{W}_{E}^{(\alpha)}
$$

If LES are used in simulations, $\overline{\mathbf{U}}_{E}$ is a always a filtered velocity but, under highly sparse conditions, we do not recommend an additional Lagrangian-scale filtering of the particle velocities.

We now give an illustration for the effect of the numerical or mixing-induced diffusion, observed in sparseLagrangian simulations under practical grid resolutions. We consider only one scalar - the mixture fraction represented by $\bar{f}_{E}=\bar{Y}_{E}$ and $Z_{f}$ with $W=0$. Since the same diffusion coefficient $D_{0}+D_{E t}$ is used in both Eulerian and Lagrangian simulations (that is $b^{i j}=\delta^{i j}\left(2 D_{0}+2 D_{E t}\right)^{1 / 2}$ in (1)), both Eulerian mixture fraction $\bar{f}_{E}$ and the particle average mixture fraction $\left\langle Z_{f}\right\rangle$ satisfy the same equation (49) [8]. In other words, without numerical diffusion induced by finite-difference nature of the Eulerian numerical scheme and mixing-induced diffusion of the Lagrangian scheme, profiles of $\bar{f}_{E}$ and $\left\langle Z_{f}\right\rangle$ must coincide. The same statement obviously applies to the mean profiles $\left\langle\bar{f}_{E}\right\rangle_{m}$ and $\left\langle Z_{f}\right\rangle_{m}$ which are fully averaged over the ensemble of turbulent fluctuations. Assuming that the averages are evaluated accurately, any observed differences between profiles of $\left\langle\bar{f}_{E}\right\rangle_{m}$ and $\left\langle Z_{f}\right\rangle_{m}$ can be attributed only to the numerical diffusion of the Eulerian scheme or mixing-induced diffusion of the Lagrangian scheme.

The simulation results shown here are for Sandia Flame E [39, 40] for which detailed experimental data are available. The details of modelling methodology, results of the simulations and overall performance of the model are discussed in other publications $[10,11,41]$. For these simulations the cylindrical computational domain, which extends 70 jet diameters in the axial direction, contains $1024 \times 55 \times 32$ cells and the smallest finite volume cells at the axis are $0.5 \mathrm{~mm} \times 0.5 \mathrm{~mm} \times \pi / 32$ radians. This grid resolution is in line with reasonable resolutions used in practical LES. The maximum ratio of subgrid to total variance in the shear layer close to the nozzle is about 30\%. The sparse-Lagrangian FDF calculations with MMC conditioning shown below use nominally only one Pope particle per 32 LES cells.

Figure 1 demonstrates mean profiles of Eulerian and Lagrangian mixture fractions close to the nozzle. As expected both profiles are consistent with each other and have a reasonable match with the experimental data. However, the same profiles, shown for another location further downstream (Figure 2), indicate that the Eulerian mixture fraction appears to be more diffusive than the Lagrangian mixture fraction. As previously discussed, this 
can be attributed only to the numerical diffusion of the Eulerian scheme. The numerical diffusion effects tend to be accumulated at downstream locations. This accumulation is less pronounced for the scalar variance, which is also shown in both figures, due to the balance of production and dissipation of the variance in the jet. Figure 3 demonstrates the same effect for axial values of the mixture fraction. The Lagrangian curve is given without smoothing to show the effect of stochastic scattering associated with averaging over the particle values.

It should be remembered that Lagrangian simulations presented here are coupled with the Eulerian LES and, of course, do not represent an autonomous method. Lagrangian simulations, however, can effectively utilise realistic topological properties of the noticeably diffusive Eulerian mixture fraction and make evaluation of the mixture fraction by Pope particles less diffusive even if these particles are used at a much lower resolution than the Eulerian grid. This example illustrates effectiveness of Pope particles (in general and with MMC conditioning in particular) for controlling numerical diffusion in low-resolution Lagrangian simulations.

\section{Conclusions}

The case of "convergence to a model" for sparse-Lagrangian FDF model is considered. The number of Pope particles (i.e. stochastic particles used in the simulations) is deemed to approach the limit of infinity in a way that maximally preserves the properties of the original sparse model. This is a conceptual consideration only as an expensive model utilising many particles but behaving like a computationally efficient sparse model can never be practical. Diffusion generated by mixing between Pope particles with non-zero spacing, which was previously seen as a source of numerical error, is now treated as a modelling tool in order to examine convergence of a particle PDF to the FDF.

The main difference between the effectively sparse conceptual model (i.e. a model populated by a large number of particles but preserving the features of the sparse model) and the truly sparse simulations is the possibility of simulating more detailed structures of the scalar fields in the former while the latter can only model equivalent statistics at the smallest scales. The main effect of introducing a large number of particles in the effectively sparse model is the disappearance of stochastic dependencies between particles. Although the analysis of the model is

simpler when velocity is filtered at the Lagrangian filter scale, we show that such Lagrangian-scale velocity filtering may have a number of detrimental effects that can bring unnecessary complications into the simulations. 


\section{Acknowledgment}

The authors thank S. B. Pope for useful remarks and acknowledge the provision of simulation results by Yipeng

Ge. This work is supported by Australian Research Council.

\section{Appendix: diffusive effects in Curl's mixing}

\subsection{Analysis of the mixing term in the PDF equation}

We now consider expansion of the PDF into the Taylor series

$$
P_{Z}\left(\mathbf{Z}_{2}^{\circ} \mid \mathbf{x}_{2}\right)=P_{Z}\left(\mathbf{Z}_{2}^{\circ} \mid \mathbf{x}_{1}\right)+\left(\frac{\partial P_{Z}\left(\mathbf{Z}_{2}^{\circ} \mid \mathbf{x}\right)}{\partial x^{i}}\right)_{1} \Delta x^{i}+\frac{1}{2}\left(\frac{\partial^{2} P_{Z}\left(\mathbf{Z}_{2}^{\circ} \mid \mathbf{x}\right)}{\partial x^{i} \partial x^{j}}\right)_{1} \Delta x^{i} \Delta x^{j}+\ldots
$$

where $\Delta x^{i}=x_{2}^{i}-x_{1}^{i}$ is introduced. This expansion corresponds to the following representations of the mixing operator

$$
\begin{gathered}
{\left[\frac{d P_{Z}}{d t}\right]_{\text {mix }}=\left[\frac{d P_{Z}}{d t}\right]_{\text {mix } 0}+\left[\frac{d P_{Z}}{d t}\right]_{\text {mix } 1}+\left[\frac{d P_{Z}}{d t}\right]_{\text {mix } 2}+\ldots} \\
{\left[\frac{d P_{Z}(\mathbf{Z} \mid \mathbf{x})}{d t}\right]_{\text {mix } 0}=\hat{\theta}\left[P_{Z}\left(\mathbf{Z}^{\circ} \mid \mathbf{x}\right) * P_{Z}\left(\mathbf{Z}^{\circ} \mid \mathbf{x}\right)\right]_{\text {mix }}} \\
{\left[\frac{d P_{Z}}{d t}\right]_{\text {mix } 1}=0} \\
{\left[\frac{d P_{Z}\left(\mathbf{Z}^{\circ} \mid \mathbf{x}\right)}{d t}\right]_{\text {mix } 2}=\frac{\hat{\theta} I^{i j}}{2}\left[P_{Z}\left(\mathbf{Z}^{\circ} \mid \mathbf{x}\right) * \frac{\partial^{2} P_{Z}\left(\mathbf{Z}^{\circ} \mid \mathbf{x}\right)}{\partial x^{i} \partial x^{j}}\right]_{\text {mix }}}
\end{gathered}
$$

where

$$
\begin{aligned}
I^{i j}=\left\langle\Delta x^{i} \Delta x^{j}\right\rangle_{V} & \equiv \frac{1}{\theta_{V}} \int \theta_{12} \Delta x^{i} \Delta x^{j} d V, \theta_{V}=\int \theta_{12} d \mathbf{x}_{2} \\
\hat{\theta} & \equiv \theta_{V}(n-1) P_{x} \approx \theta_{V} \rho_{n}
\end{aligned}
$$

since $n-1 \approx n$ for large $n$. The first order term in the expansion vanishes as the mixing volume is selected so that $x_{1}$ is located at its mass centre. Using the following identity

$$
P_{Z} \frac{\partial^{2} P_{Z}}{\partial x^{i} \partial x^{j}}=\frac{\partial}{\partial x^{i}}\left(P_{Z} \frac{\partial P_{Z}}{\partial x^{j}}\right)-\frac{\partial P_{Z}}{\partial x^{i}} \frac{\partial P_{Z}}{\partial x^{j}}
$$

The second term in the expansion becomes

$$
\left[\frac{d P_{Z}}{d t}\right]_{\text {mix } 2}=\underbrace{\frac{\partial}{\partial x^{i}}\left(\frac{\hat{\theta} I^{i j}}{2}\left[P_{Z} * \frac{\partial P_{Z}}{\partial x^{j}}\right]_{\text {mix }}\right)}_{\text {mix } 2 \mathrm{D}}-\underbrace{\frac{\hat{\theta} I^{i j}}{2}\left[\frac{\partial P_{Z}}{\partial x^{i}} * \frac{\partial P_{Z}}{\partial x^{j}}\right]_{\text {mix }}}_{\text {mix2N }}
$$


Here, we place $\hat{\theta} I^{i j}$ under differentiation sign since the divergent form of the term "mix $2 \mathrm{D}$ " is consistent with the conservation property

$$
\int\left[\frac{d\left\langle Z^{(\alpha)}\right\rangle}{d t}\right]_{\text {mix }} d \mathbf{x}=0
$$

of Curl's mixing. Note that the term "mix0" in (52) and, as shown below, the term "mix2N" in (58) satisfy constraint (17) and, consequently, the condition specified by (59). Since Curl's mixing is compliant with (59), so must be the term "mix2D" but in its originally derived form (with $\hat{\theta} I^{i j}$ placed outside the differentiation sign), this term is not consistent with (59). The explanation for this lays in the fact that we consider only a truncated representation of Curl's mixing in (51) and this non-compliance factor is hidden in the higher order terms. These terms were neglected in our analysis. The physical reason for this is that the parameters of Curl's mixing are the same for any mixing couple and, thus, have limited variations within the mixing volume while there are no similar

restrictions imposed on values $Z_{1}$ and $Z_{2}$. Essentially, we neglect gradients of $\hat{\theta} I^{i j}$ in comparison with gradients of $Z$. Hence, it is reasonable for us to reinstate the conservation property (59) by making a higher order adjustment to the equation and moving $\hat{\theta} I^{i j}$ under the differentiation sign in the term "mix $2 \mathrm{D}$ ".

Evaluation of the first moment of the term "mix $2 \mathrm{~N}$ " yields

$$
\begin{aligned}
{\left[\frac{d\left\langle Z^{(\alpha)}\right\rangle}{d t}\right]_{\text {mix } 2 \mathrm{~N}}=} & \frac{\hat{\theta} I^{i j}}{2}\langle\tilde{\eta}\rangle \int\left(Z_{2}^{(\alpha)}-Z_{1}^{(\alpha)}\right) \frac{\partial P_{Z}\left(\mathbf{Z}_{1} \mid \mathbf{x}\right)}{\partial x^{i}} \frac{\partial P_{Z}\left(\mathbf{Z}_{2} \mid \mathbf{x}\right)}{\partial x^{j}} d \mathbf{Z}_{1} d \mathbf{Z}_{2}= \\
= & \frac{\hat{\theta} I^{i j}}{2}\langle\tilde{\eta}\rangle\left(\frac{\partial\left\langle Z^{(\alpha)}\right\rangle}{\partial x^{i}}-\frac{\partial\left\langle Z^{(\alpha)}\right\rangle}{\partial x^{j}}\right)=0
\end{aligned}
$$

due to symmetry of the tensor $I^{i j}$. Assuming that the mixing volume is spherical and that density variations within this volume are not significant, we obtain $I^{i j}=\delta^{i j} I$ where $I=\hat{r}_{1}^{2}$ is determined by the radius of the mixing volume.

\subsection{Evaluation of the first and second moments}

The moments of Curl's mixing are evaluated according to equations

$$
\begin{gathered}
{\left[\frac{\partial\left\langle Z_{1}\right\rangle}{\partial t}\right]_{\text {mix }}=\hat{\theta}\langle\tilde{\eta}\rangle\left\langle Z_{2}-Z_{1}\right\rangle} \\
{\left[\frac{\partial\left\langle Z_{1}^{2}\right\rangle}{\partial t}\right]_{\text {mix }}=2 \hat{\theta}\langle\tilde{\gamma}\rangle\left\langle Z_{1} Z_{2}-Z_{1}^{2}\right\rangle+\hat{\theta}\left\langle\tilde{\eta}^{2}\right\rangle\left\langle Z_{2}^{2}-Z_{1}^{2}\right\rangle}
\end{gathered}
$$

so that the effect of mixing on variance takes the form

$$
\left[\frac{\partial\left\langle z_{1}^{2}\right\rangle}{\partial t}\right]_{\text {mix }}=\hat{\theta}\left(-2\langle\tilde{\gamma}\rangle\left\langle z_{1}^{2}\right\rangle+\left\langle\tilde{\eta}^{2}\right\rangle\left\langle z_{2}^{2}-z_{1}^{2}\right\rangle+\left\langle\tilde{\eta}^{2}\right\rangle\left(\left\langle Z_{2}\right\rangle-\left\langle Z_{1}\right\rangle\right)^{2}\right)
$$


where $\tilde{\gamma} \equiv \tilde{\eta}-\tilde{\eta}^{2}$. The superscript " $(\alpha)$ " is omitted here and further in the subsection. With the use of Taylor series expansions

$$
\begin{aligned}
& \left\langle Z_{2}\right\rangle=\left\langle Z_{1}\right\rangle+\left(\frac{\partial\langle Z\rangle}{\partial x^{i}}\right)_{1} \Delta x^{i}+\frac{1}{2}\left(\frac{\partial^{2}\langle Z\rangle}{\partial x^{i} \partial x^{j}}\right)_{1} \Delta x^{i} \Delta x^{j}+\ldots \\
& \left\langle z_{2}^{2}\right\rangle=\left\langle z_{1}^{2}\right\rangle+\left(\frac{\partial\left\langle z^{2}\right\rangle}{\partial x^{i}}\right)_{1} \Delta x^{i}+\frac{1}{2}\left(\frac{\partial^{2}\left\langle z^{2}\right\rangle}{\partial x^{i} \partial x^{j}}\right)_{1} \Delta x^{i} \Delta x^{j}+\ldots
\end{aligned}
$$

we obtain

$$
\begin{gathered}
{\left[\frac{\partial\left\langle Z_{1}\right\rangle}{\partial t}\right]_{\text {mix }}=\frac{\hat{\theta}\langle\tilde{\eta}\rangle I^{i j}}{2}\left(\frac{\partial^{2}\langle Z\rangle}{\partial x^{i} \partial x^{j}}\right)_{1}+\ldots} \\
{\left[\frac{\partial\left\langle z_{1}^{2}\right\rangle}{\partial t}\right]_{\text {mix }}=-2\langle\tilde{\gamma}\rangle \hat{\theta}\left\langle z_{1}^{2}\right\rangle+\frac{\hat{\theta}\left\langle\tilde{\eta}^{2}\right\rangle I^{i j}}{2}\left(\frac{\partial^{2}\left\langle z^{2}\right\rangle}{\partial x^{i} \partial x^{j}}+2 \frac{\partial\langle Z\rangle}{\partial x^{i}} \frac{\partial\langle Z\rangle}{\partial x^{j}}\right)_{1}}
\end{gathered}
$$

The first order terms disappear since as we assume that particle "1" is located in the centre of the mixing volume. Finally, we invoke the same arguments as we did for the term "mix2D" in (58) and place the term $\hat{\theta} I^{i j}$ under the differentiation sign of the diffusion-type terms. The last equations can now be rewritten as

$$
\begin{gathered}
{\left[\frac{\partial\left\langle Z_{1}\right\rangle}{\partial t}\right]_{\text {mix }}=\frac{\partial}{\partial x^{i}}\left(\frac{\hat{\theta}\langle\tilde{\eta}\rangle I^{i j}}{2} \frac{\partial\langle Z\rangle}{\partial x^{j}}\right)+\ldots} \\
{\left[\frac{\partial\left\langle z^{2}\right\rangle}{\partial t}\right]_{\text {mix }}=-\hat{\theta}\langle\tilde{\gamma}\rangle\left(2\left\langle z^{2}\right\rangle+I^{i j} \frac{\partial\langle Z\rangle}{\partial x^{i}} \frac{\partial\langle Z\rangle}{\partial x^{j}}\right)+\frac{\partial}{\partial x^{i}}\left(\frac{\hat{\theta}\left\langle\tilde{\eta}^{2}\right\rangle I^{i j}}{2} \frac{\partial\left\langle z^{2}\right\rangle}{\partial x^{j}}\right)+\hat{\theta}\langle\tilde{\eta}\rangle I^{i j} \frac{\partial\langle Z\rangle}{\partial x^{i}} \frac{\partial\langle Z\rangle}{\partial x^{j}} .}
\end{gathered}
$$

If the mixing volume possesses spherical symmetry then $I^{i j}=\delta^{i j} I$. Assuming that $\theta_{12}$ is given by $\theta_{12}=\theta_{0}$ for $r^{2} \equiv \Delta x^{i} \Delta x^{i} \leq r_{m}^{2}$ and $\theta_{12}=0$ for $r>r_{m}$, we obtain $I=\hat{r}_{1}^{2}$ where $\hat{r}_{1}^{2}=r_{m}^{2} / 5$ in a three-dimensional space [8].

\section{References}

[1] S. B. Pope, Computations of turbulent combustion: progress and challenges, Proc. Combust. Inst 23 (1990) $591-612$.

[2] F. Gao, E. E. O’Brien, A large-eddy simulation scheme for turbulent reacting flows, Phys. Fluids A 5 (1993) $1282-1284$.

[3] P. J. Colucci, F. A. Jaberi, P. Givi, S. B. Pope, Filtered density function for large eddy simulation of turbulent reacting flows, Phys. Fluids 10 (1998) 499-515. 
[4] F. A. Jaberi, P. J. Colucci, S. James, P. Givi, S. B. Pope, Filtered mass density function for large-eddy simulation of turbulent reacting flows, J. Fluid Mech. 401 (1999) 85-121.

[5] S. B. Pope, Pdf methods for turbulent reactive flows, Prog. Energy Combust. Sci. 11 (1985) 119-192.

[6] R. S. Rogallo, P. Moin, Ten questions concerning the large-eddy simulation of turbulent flows, Ann. Rev. Fluid Mech. 16 (1984) 99-137.

[7] S. B. Pope, Ten questions concerning the large-eddy simulation of turbulent flows, New Journal of Physics 6 (2004) 35 .

[8] A. Y. Klimenko, Lagrangian particles with mixing. I. simulating scalar transport, Phys. Fluids 21 (2009) 065101.

[9] A. Klimenko, Why does one need to be taught engineering logic?, in: International Conference on Engineering Education (ICEE), Portugal, 2007, paper 574.

[10] M. J. Cleary, A. Y. Klimenko, A generalised multiple mapping conditioning approach for turbulent combustion, Flow, Turbulence and Combustion 82 (2009) 477-491.

[11] M. J. Cleary, A. Y. Klimenko, J. Janicka, M.Pfitzner, A sparse-lagrangian multiple mapping conditioning model for turbulent diffusion flames, in: Proc. Combust. Inst., Vol. 32, 2009, p. accpeted for publication.

[12] J. Meyers, B. Geurts, P. Sagaut, A computational error-assessment of central finite-volume discretizations in large-eddy simulation using a Smagorinsky model, Journal of Computational Physics 227 (2007) 156173.

[13] B. Geurts, LES quality assessment, in: Ninth International Workshop on Measurement and Computation of Turbulent Nonpremixed Flames (TNF-9), 2008, p. 10.

[14] A. Y. Klimenko, Lagrangian particles with mixing. II. sparse-lagrangian methods in application for turbulent reacting flows, Phys. Fluids 21 (2009) 065102.

[15] T. D. Dreeben, S. B. Pope, Probability density function and Reynolds-stress modeling of near-wall turbulent flows, Phys. Fluids 9 (1997) 154-163.

[16] M. S. Anand, S. B. Pope, H. C. Mongia, A PDF method for turbulent recirculating flows, in: Lecture Notes in Engineering, Vol. 40, Springer-Verlag, 1989, pp. 672-693. 
[17] V. R. Kuznetsov, V. A. Sabelnikov, Turbulence and Combustion, Hemisphere, New York, 1990.

[18] N. Peters, Turbulent Combustion, Cambridge University Press, 2000.

[19] A. Y. Klimenko, R. W. Bilger, Conditional moment closure for turbulent combustion, Prog. Energy Combust. Sci. 25 (1999) 595-687.

[20] A. Y. Klimenko, S. B. Pope., A model for turbulent reactive flows based on multiple mapping conditioning., Physics of Fluids 15 (2003) 1907-1925.

[21] C. Dopazo, Recent developments in Pdf methods, in: P. A. Libby, F. A. Williams (Eds.), Turbulent Reacting Flows, Academic Press, London, 1994, Ch. 7, pp. 375-474.

[22] S. B. Pope, Turbulent Flows, Cambridge University Press, Cambridge, 2000.

[23] R. Fox, Computational Models for Turbulent Reacting Flows, Cambridge University Press, Cambridge, 2003.

[24] S. Heinz, Statistical Mechanics of Turbulent Flows, Springer, Berlin, 2003.

[25] H. Pitsch, Large-Eddy simulations of turbulent combustion, Annu. Rev. Fluid Mech. 38 (2006) 453-482.

[26] D. Haworth, Progress in probability density function methods for turbulent reacting flows, Prog. Energy Combust. Sci. 36 (2010) 168-259.

[27] B. Sawford, Micro-mixing modelling of scalar fluctuations for plumes in homogeneous turbulence, Flow, Turbulence and Combustion 72 (2004) 133-160.

[28] A. Klimenko, On simulating scalar transport by mixing between Lagrangian particles, Phys. Fluids 19 (2007) 031702.

[29] V. Raman, H. Pitsch, R. O. Fox, Hybrid large-eddy simulation/lagrangian filtered-density-function approach for simulating turbulent combustion, Combust. Flame 143 (2005) 56-78.

[30] R. L. Curl, Dispersed phase mixing. I., AIChE J. 9 (1963) 175.

[31] J. Janicka, W. Kolbe, W. Kollmann, Closure of the transport equation for the probability density function of turbulent scalar fields, J. Non-Equilib. Thermodyn 4 (1977) 47-66. 
[32] A. Y. Klimenko, Matching conditional moments in pdf modelling of non-premixed combustion, Combust. Flame 143 (2005) 369-385.

[33] A. W. Cook, J. J. Riley, A subgrid model for equilibrium chemistry in turbulent flows, Phys. Fluids 6 (1994) $2868-2870$.

[34] C. Jimenez, F. Ducros, B. Cuenot, B. Bedat, A subgrid model for equilibrium chemistry in turbulent flows, Phys. Fluids 13 (2001) 1748-1754.

[35] G. K. Batchelor, I. D. Howells, A. A. Townsend, Small-scale variation of convected quantities like temperature in turbulent fluid. Part 2: The case of large conductivity, J. Fluid Mech. 113 (1959) 134-139.

[36] A. P. Wandel, A. Y. Klimenko, Testing MMC mixing for Monte-Carlo PDF simulations, Phys. Fluids 17 (2005) 128105.

[37] K. R. Sreenivasan, Possible effects of small-scale intermittency in turbulent reacting flows, Flow, Turbulence and Combustion 72 (2004) 115-131.

[38] A. Kronenburg, M. J. Cleary, Multiple mapping conditioning for flames with partial premixing combustion and flame, Combust. Flame 155 (2008) 215-231.

[39] R. S. Barlow, J. Frank, Effects of turbulence on species mass fractions in methane/air flames, in: Proc. Combust. Inst., Vol. 27, 1998, pp. 1087-1095.

[40] C. Schneider, A. Dreizler, J. Janicka, E. P. Hassel, Flow field measurements of stable and locally extinguishing hydrocarbon-fuelled jet flames, Combust. Flame 135 (2003) 185-190.

[41] Y.Ge, M. Cleary, A. Klimenko, Sparse-lagrangian fdf simulations of sandia flame e with density coupling, in: Proc. Combust. Inst., Vol. 33, 2010, accepted. 


\section{FIGURE CAPTIONS}

Figure 1: Radial profiles of average and rms mixture fraction in Sandia Flame E at the axial distance of one

nozzle diameter. Lines adorned with closed symbols are for Eulerian LES while unadorned lines are for the sparseLagrangian FDF. Open triangular symbols represent experimental data [39, 40].

Figure 2: Radial profiles at 30 nozzle diameters. The simulation case and notations are the same as in Figure 1.

Figure 3: Axial profiles versus normalised distance from the nozzle. The simulation case and notations are the same as in Figure 1. 


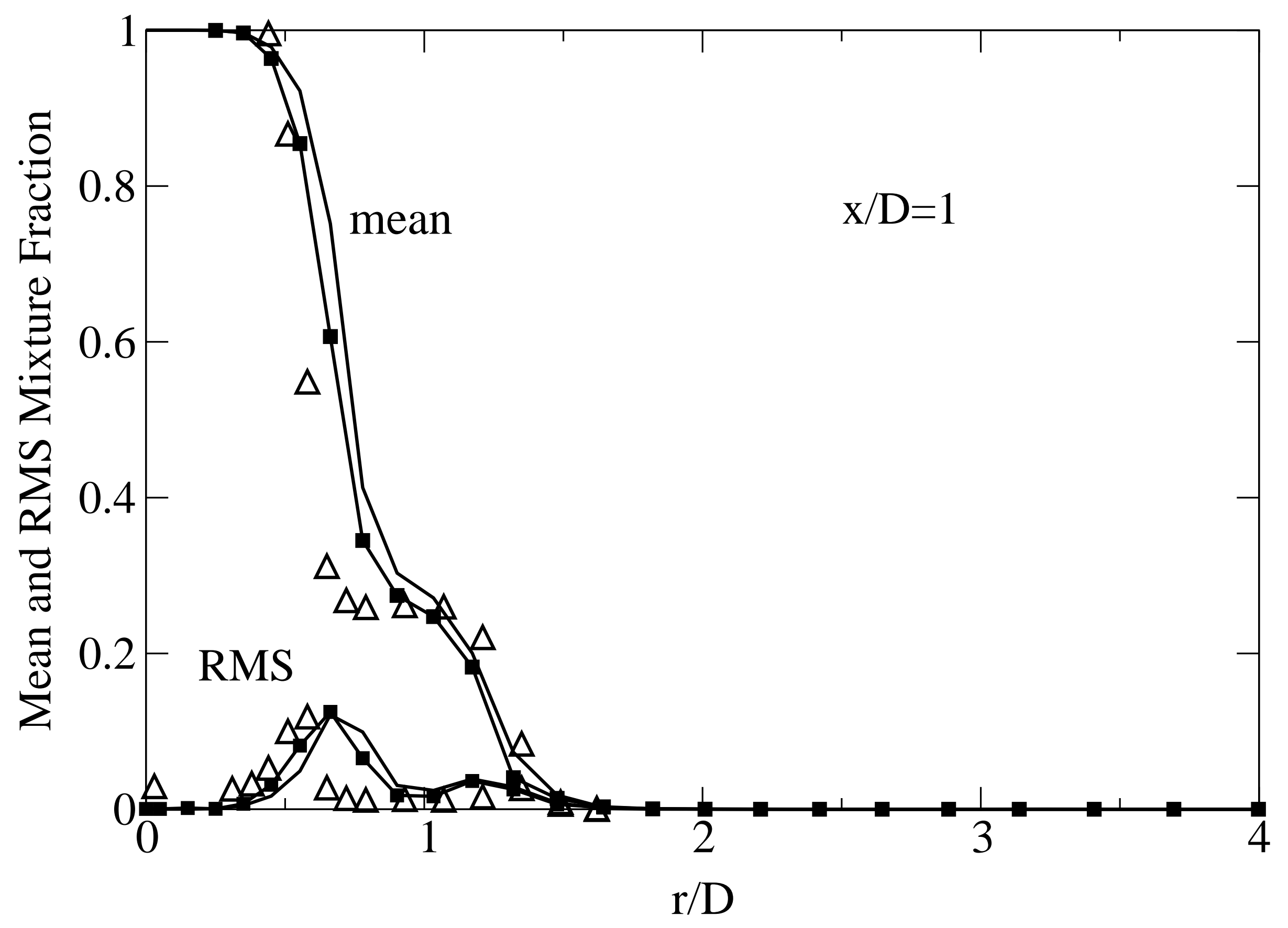




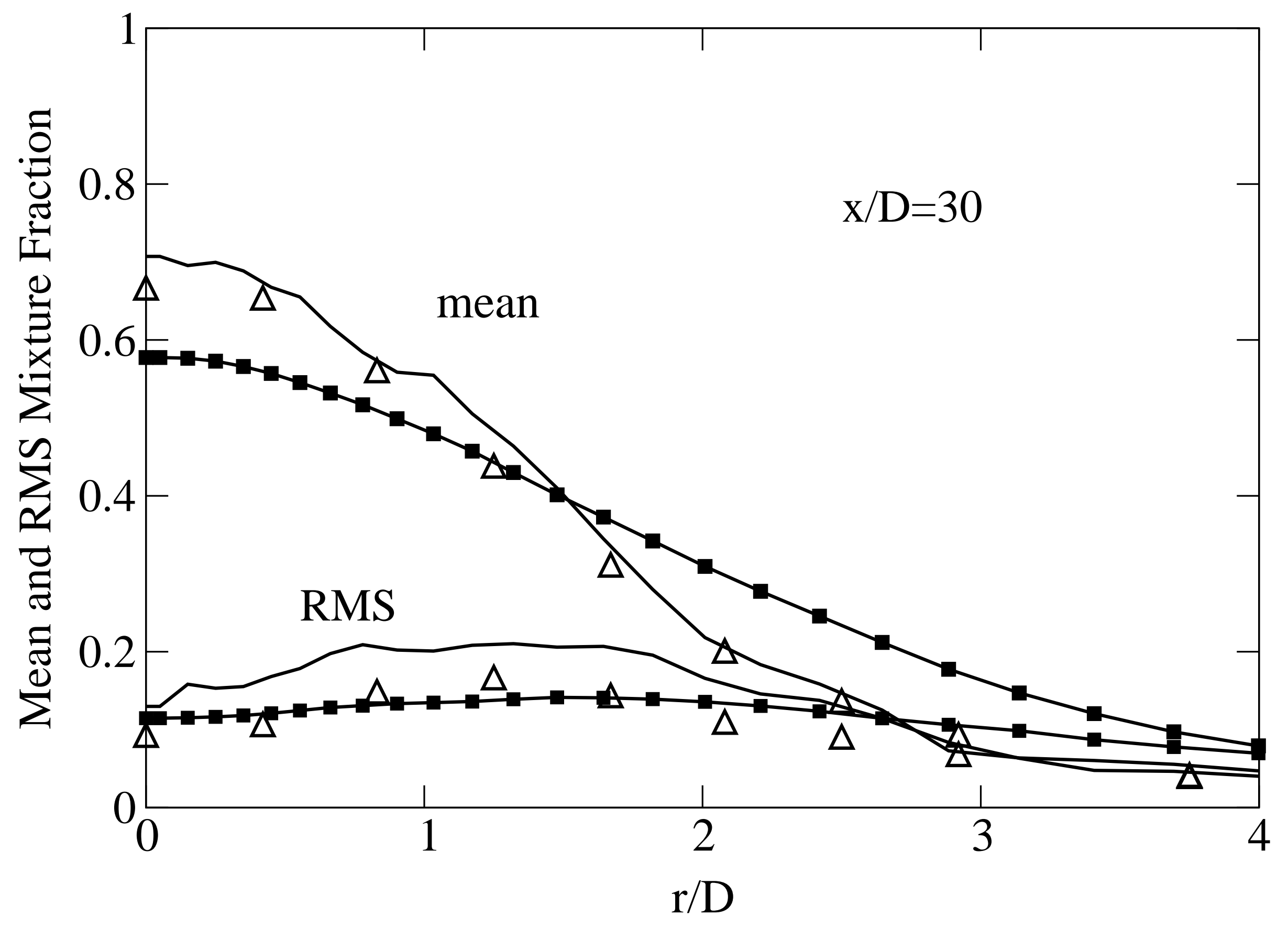




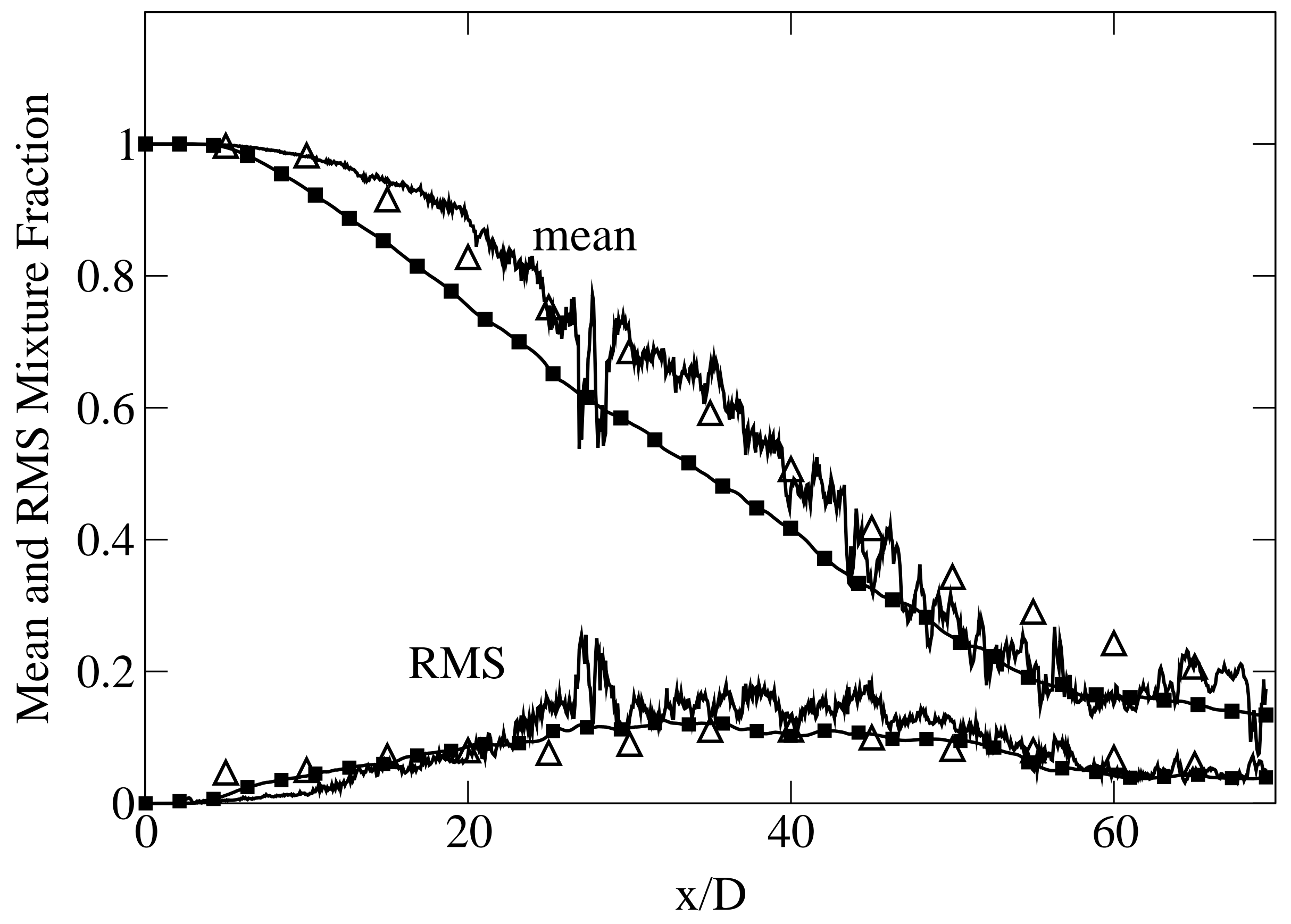

